\title{
Enhanced light element imaging in atomic resolution scanning transmission electron microscopy
}

\author{
S. D. Findlay ${ }^{\mathrm{a}}$, Y. Kohno ${ }^{\mathrm{b}}$, L.A. Cardamone ${ }^{\mathrm{a}}$, Y. Ikuhara $^{\mathrm{c}, \mathrm{de}, \mathrm{e}}$, N. Shibatac, ${ }^{\mathrm{c} f}$ \\ ${ }^{a}$ School of Physics, Monash University, Victoria 3800, Australia \\ ${ }^{b}$ JEOL Ltd., Tokyo 196-8558, Japan \\ ${ }^{c}$ Institute of Engineering Innovation, School of Engineering, University of Tokyo, Tokyo 113-8656, Japan \\ ${ }^{d}$ Nanostructures Research Laboratory, Japan Fine Ceramics Center, Nagoya 456-8587, Japan \\ ${ }^{e}$ WPI Advanced Institute for Materials Research, Tohoku University, Sendai 980-8577, Japan

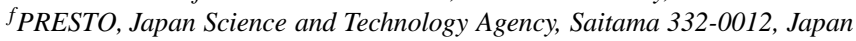

\begin{abstract}
We show that an imaging mode based on taking the difference between signals recorded from the bright f eld (forward scattering region) in atomic resolution scanning transmission electron microscopy provides an enhancement of the detectability of light elements over existing techniques. In some instances this is an enhancement of the visibility of the light element columns relative to heavy element columns. In all cases explored it is an enhancement in the signal-to-noise ratio of the image at the light column site. The image formation mechanisms are explained and the technique is compared with earlier approaches. Experimental data, supported by simulation, are presented for imaging the oxygen columns in $\mathrm{LaAlO}_{3}$. Case studies looking at imaging hydrogen columns in $\mathrm{YH}_{2}$ and lithium columns in $\mathrm{Al}_{3} \mathrm{Li}$ are also explored through simulation, particularly with respect to the dependence on defocus, probe-forming aperture angle and detector collection aperture angles.
\end{abstract}

Keywords:

scanning transmission electron microscopy (STEM), atomic resolution imaging, annular bright $f$ eld (ABF).

\section{Introduction}

The scientif $\mathrm{c}$ and technological importance of light elements in a diverse range of materials - oxygen in cuprate superconductors, lithium in battery materials, hydrogen in hydrogen-storage materials, to name but three - makes imaging of these elements highly desirable in materials characterization. For atomic resolution structure analysis, annular dark f eld (ADF) imaging in scanning transmission electron microscopy (STEM) has long provided robust and directly interpretable images, but the thermal-scattering-dominated contrast strongly favours heavy elements [1]. ADF has recently been complemented by the development of annular bright f eld (ABF) imaging [2-4], involving an annular detector in the outer area of the bright $f$ eld (forward scattering region) and enabling the simultaneous imaging of light and heavy elements with similar contrast. A similarly recent and closely related approach, middle-angle bright 
f eld (MaBF) ${ }^{1}$ imaging [5], involves an on-axis disk detector with outer angle about half (hence "middle") the probeforming aperture angle and shows light and heavy elements with opposite contrast but consequently better resolution. $\mathrm{MaBF}$ and $\mathrm{ADF}$ images can be combined into colour composite images to aid visual interpretation [6].

Though not without precedent in the earlier literature, the usage of an annular detector in or near the bright feld having been much explored [7-9], the newfound appreciation that for atomically f ne probes ABF imaging enabled robust visualization of light elements over a wide thickness range led to its rapid uptake through the feld [10-21]. The direct ABF imaging of lithium [22-30] and of hydrogen [31,32] within crystalline matrices using STEM is of particular note because of the technological interest in lithium-bearing materials for lithium-battery devices and hydrogen-bearing materials for hydrogen storage applications. Nevertheless, $\mathrm{ABF}$ of these very light elements remains challenging. Light-element-bearing specimens tend to damage under the electron beam, but trying to avoid this by reducing the dose reduces the signal-to-noise ratio. Moreover, ABF imaging of very light elements anyway involves looking for a small signal, a slight intensity dip, which may be of comparable magnitude to scattering artefacts or to noise, on a large background. Techniques which improve the signal, either by enhancing the signal in an absolute sense or by minimizing the dose via recording as many scattered electrons as possible [33], will be an asset to materials characterization. In this paper we show that the difference between the $\mathrm{ABF}$ and MaBF images, which for convenience we refer to as enhanced $\mathrm{ABF}$ (eABF) imaging, is such a technique.

\section{Conceptual background}

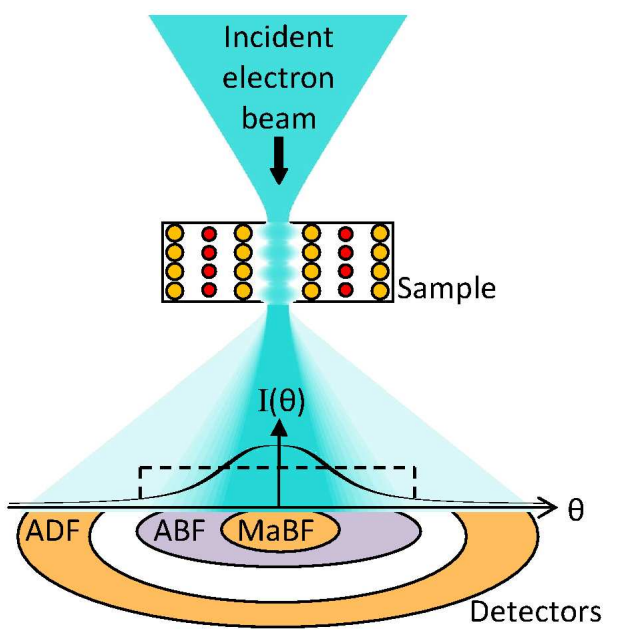

Figure 1: Schematic of some key detector geometries in atomic resolution STEM.

\footnotetext{
${ }^{1}$ We have taken the liberty of using a lower case "a" in MaBF to emphasise that it does not abbreviate the same word as the "A" in ABF and ADF.
} 
It is useful to $\mathrm{f}$ rst develop an intuitive understanding of $\mathrm{ABF}$ and MaBF imaging. Fig. 1 shows a schematic of the electron probe "channelling" (scattering) along a column of atoms and the resultant intensity prof le in the diffraction pattern as a function of angle $\theta$ away from the optical axis. In the absence of a sample, the diffraction prof le would be a top-hat function (dashed line) spanning the bright $f$ eld. Scattering through a sample redistributes this intensity (solid line). The real intensity distribution would likely have $\mathrm{f}$ ner structural features, but the general behaviour-the intensity increased in the inner area of the bright f eld, reduced in the outer area of the bright $f$ eld, and spreading out into the dark f eld - are correct for atomically f ne probes atop relatively light columns. Atop heavier columns, a larger portion of electrons are scattered into the dark f eld, reducing the intensity throughout the bright f eld. That both mechanisms lead to a reduced intensity in the outer area of the bright f eld over a wide range of elements and sample thicknesses is the basis of the robust absorptive form of ABF images. That the intensity tends to peak up in the forwards direction for light element columns (where channelling dominates over thermal scattering) but reduces for heavy element columns (where thermal scattering dominates over channelling) is the basis for the mixed-contrast form of MaBF images. A more rigorous derivation of this behaviour on an individual column basis and using the s-state model [34] has been given by Findlay and colleagues [3,4] and a full Bloch state analysis for MaBF imaging has been given by Ohtsuka et al. [5].

By way of example, ADF, ABF and MaBF images of $\mathrm{LaAlO}_{3}$ viewed along the [001] direction as a function of specimen thickness are compared in Figs. 2(a)-(c) (parameters are given in the f gure caption; structural data taken from Ref. [35]). For each mode, the top half of the panel assumes an inf nitesimal/point effective source size (i.e. perfect spatial coherence) while the lower half, more realistically, assumes a f nite, incoherent effective source described via a Gaussian of half-width $0.05 \mathrm{~nm}$ [36], both, of course, subjected to the diffraction limit of the probeforming lens. As expected, the ADF images in Fig. 2(a) are dominated by the heavy La column, with the Al/O column faintly visible but the $\mathrm{O}$ columns invisible. The $\mathrm{ABF}$ images assuming perfect spatial coherence, the top half of Fig. 2(b), show all columns $-\mathrm{La}, \mathrm{Al} / \mathrm{O}$ and pure $\mathrm{O}-$ consistent with the previously touted utility of $\mathrm{ABF}$ for imaging light elements. However, when spatial incoherence is taken into account in the bottom half of Fig. 2(b), the $\mathrm{O}$ columns are only weakly visible for a small subset of specimen thicknesses. The MaBF images assuming perfect spatial coherence, the top half of Fig. 2(c), show pure O columns bright and La columns dark, while the contrast of the $\mathrm{Al} / \mathrm{O}$ column is thickness dependent, sometimes with donut-shaped contrast. This mean that MaBF images are not so readily interpretable, although with simultaneous $\mathrm{ADF}$ and/or ABF images for reference this presents no great ambiguity. When spatial incoherence is taken into account, the bottom half of Fig. 2(c), some of the anomalous features are suppressed. However, the $\mathrm{O}$ columns are not washed out to as great an extent as they were in the ABF images.

A better quantitative sense of the peak heights and trough depths can be obtained from Figs. 2(d)-(g), which show line prof les through the alternating La-O-La column sequence (Figs. 2(d)-perfect spatial coherence and (f)incoherent effective source) and through the alternating $\mathrm{O}-\mathrm{Al} / \mathrm{O}-\mathrm{O}$ column sequence (Figs. 2(e)-perfect spatial coherence and (g)-incoherent effective source) assuming a $20 \mathrm{~nm}$ thick sample. The $\mathrm{La}-\mathrm{O}-\mathrm{La}$ column sequence is 

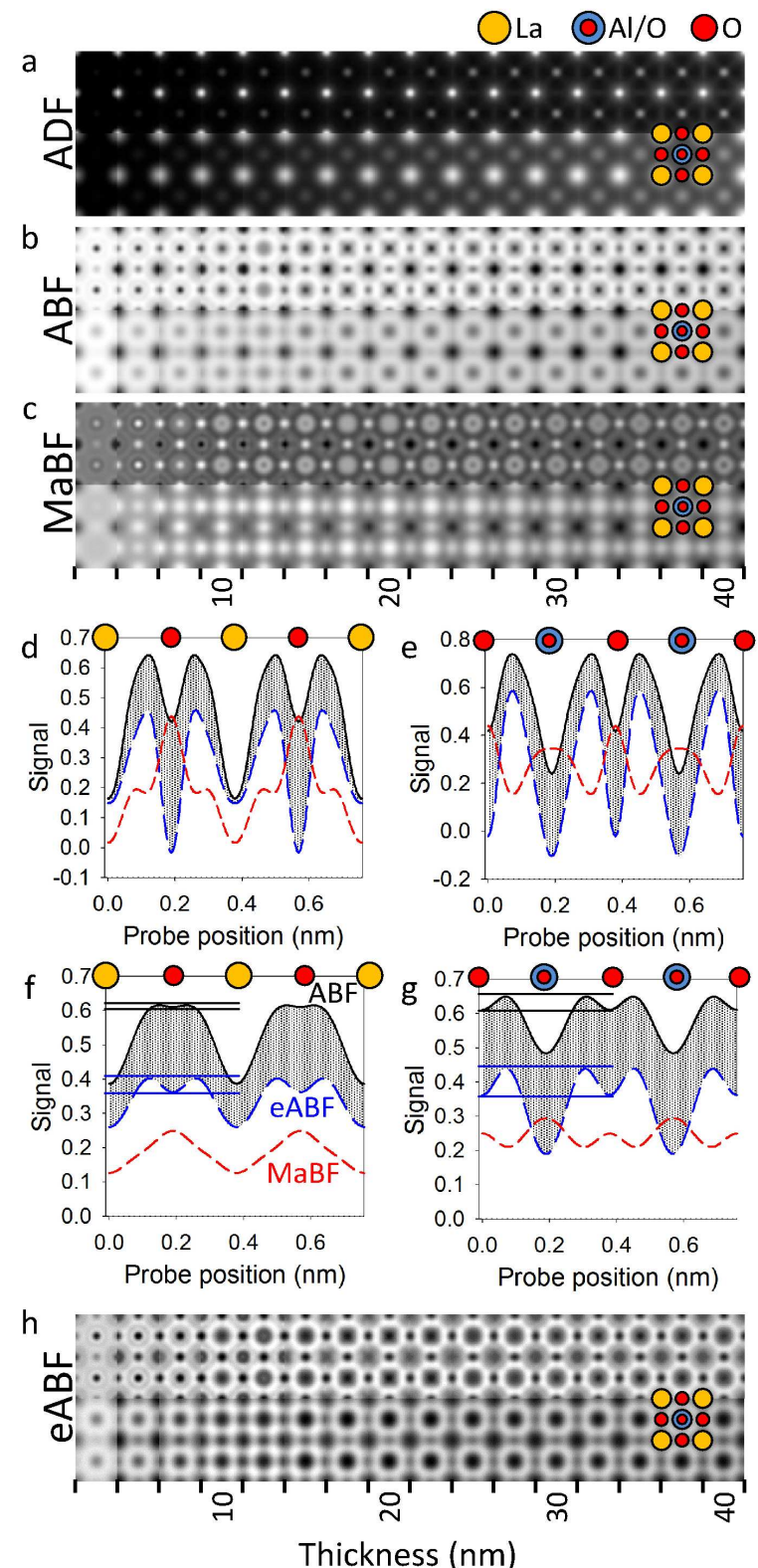

Figure 2: (a) $\mathrm{ADF}$, (b) $\mathrm{ABF}$ and (c) $\mathrm{MaBF}$ images as a function of thickness for $\mathrm{LaAlO}_{3}$ viewed along the [001] direction for a $200 \mathrm{keV}$ probe with a $23 \mathrm{mrad}$ probe-forming aperture angle. The detector ranges are: ADF 81-228 mrad; ABF 11.5-23 mrad; MaBF 0-11.5 mrad. The upper half of each panel assumes perfect spatial coherence, while the lower half incorporates a Gaussian incoherent effective source of half-width $0.05 \mathrm{~nm}$. (d) and (e) show line prof les, assuming a $20 \mathrm{~nm}$ thick sample, through the alternating $\mathrm{La}-\mathrm{O}-\mathrm{La}$ column sequence and the alternating $\mathrm{O}-\mathrm{Al} / \mathrm{O}-\mathrm{O}$ column sequence, respectively, for perfect coherence, while (f) and (g) show the same prof les assuming a Gaussian incoherent effective source of half-width $0.05 \mathrm{~nm}$. The grey shading between the ABF and eABF prof les emphasises the relative enhancement of the visibility in the pure $O$ columns in the latter over the former. The horizontal lines in (f) and (g) provide a guide to the eye of the change in local visibility of the $\mathrm{O}$ column between $\mathrm{ABF}$ and $\mathrm{eABF}$ images. (h) eABF images, being the difference between the images in (b) and (c). 
particularly instructive. In the $\mathrm{ABF}$ image, both the La and the $\mathrm{O}$ columns appear as troughs, but the shallower $\mathrm{O}$ column troughs are more severely impacted by the incoherent effective source size blurring. While for the perfectly coherent source, Fig. 2(d), the ABF signal (black line) has the $O$ columns clearly visible at almost half the depth of the heavy La columns, inclusion of incoherent effective source size renders the $\mathrm{O}$ column contrast all but invisible. $\mathrm{MaBF}$ is seen to be more robust in this sense: the La and the $\mathrm{O}$ columns appear with opposite contrast $-\mathrm{O}$ is bright while La is dark - and, though both are affected by incoherent effective source size, the broad qualitative behaviour is unchanged. In particular, the $\mathrm{O}$ peaks are still clearly peaks.

MaBF appears better at preserving reliably interpretable peaks upon the lighest element columns. If the technique has a drawback, it is the potential for ambiguous contrast for intermediate weight elements: if heavy elements appear dark and light elements appear bright, then intermediate weight elements can appear with uninformatively intermediate contrast. (Kotaka's colour composite approach overcomes this by using the simultaneously acquired ADF image to $\mathrm{f} 11$ in any gaps in the MaBF interpretation [6].) ABF maintains consistent interpretability of column contrast (excluding signif cant defocus variation applied to very thin crystals [37]), but the contrast of very light elements, especially in the presence of a large incoherent effective source size, can be weak. We wish to combine the strengths of both these imaging modes into a single imaging mode.

The reasonable $\mathrm{O}$ visibility in the $\mathrm{MaBF}$ image can be used to enhance the weak $\mathrm{O}$ visibility in the $\mathrm{ABF}$ image by taking the difference between the two. The MaBF signal is necessarily positive. Taking the difference between the $\mathrm{ABF}$ and $\mathrm{MaBF}$ signals thus lowers the signal relative to standard $\mathrm{ABF}$. (We note in passing, though it is of little consequence, that taking the difference produces a negative number when the MaBF signal exceeds the ABF signal.) The MaBF signal is largest on the $\mathrm{O}$ columns and smallest on the La columns. Taking the difference between the $\mathrm{ABF}$ and MaBF signals thus lowers the signal at the $\mathrm{O}$ sites more than it lowers the signal at the La sites, enhancing the relative visibility of $\mathrm{O}$ compared with La. The eABF $=\mathrm{ABF}-\mathrm{MaBF}$ signal is shown in Fig. 2(h) and as the red line on the plots in Figs. 2(d)-(g). The O visibility in the eABF images, Fig. 2(h), is clearly better than that in the ABF images, Fig. 2(b), even in the presence of incoherent effective source size. The grey shading between the ABF and eABF signals on the plots in Figs. 2(d)-(g) serves as a visual guide, emphasising that the signal on the O column, its trough depth, is increased relative to that at the La column by the eABF construction. Because the $\mathrm{Al} / \mathrm{O}$ column also appears as a bright peak in MaBF, the eABF construction enhances its contrast relative to that of the La column too. In the case of perfect spatial coherence, Fig. 2(e), the O column peak in the MaBF signal is larger than that of the $\mathrm{Al} / \mathrm{O}$ column, and there is an enhancement of the $\mathrm{O}$ column visibility relative to that of the $\mathrm{Al} / \mathrm{O}$ column in eABF. However, the $\mathrm{Al} / \mathrm{O}$ column is broader, and when incoherent effective source size is taken into account, Fig. $2(\mathrm{~g})$, the $\mathrm{O}$ column peak may be weaker than the $\mathrm{Al} / \mathrm{O}$ column peak, affording little enhancement of the O column relative to the $\mathrm{Al} / \mathrm{O}$ column. Finally, note that the horizontal lines in Figs. 2(f) and (h) provide a visual guide of the absolute, local visibility enhancement of the $\mathrm{O}$ column. That the trough depth is increased in eABF as compared to ABF means the visibility of the $\mathrm{O}$ column increases in an absolute sense. The derived eABF image inherits the in-quadrature addition of the noise of the ABF and MaBF images. However, as we shall presently show in detail, the visibility of the light 
elements is increased to a greater degree than the noise, and so eABF imaging provides a signal-to-noise ratio (SNR) enhancement for the local image visibility of the lightest of elements. We consider this to be its greatest merit.

\section{3. eABF imaging: key ideas and proof-of-principle experiment}

A little ref ection on the above discussion on $\mathrm{ABF}$ and MaBF imaging reveals we can expect quite generally that the $\mathrm{ABF}$ difference image will enhance the contrast of light elements relative to heavy elements. MaBF images are positive def nite, so the strong MaBF signal from light elements enhances their visibility signif cantly whereas the weak MaBF signal from heavy elements gives little-to-no enhancement of their visibility. Light element visibility is thereby preferentially enhanced. Intermediate weight columns may or may not be enhanced, but their robust visibility from the $\mathrm{ABF}$ images generally persists in the $\mathrm{eABF}$ images. The $\mathrm{ABF}$ imaging mode thus produces an image with greater visibility for the light elements than $\mathrm{ABF}$ and, unlike MaBF, with consistently interpretable contrast.

What we are really doing for light elements is taking advantage of the separate detectors to reinforce the signal: because total intensity is conserved, an intensity gain in the MaBF detector (inner area of the bright $f$ eld) implies a corresponding intensity loss in the $\mathrm{ABF}$ detector (outer area of the bright f eld). Taking the difference between these two images thus increases the signal strength. The noise, adding in quadrature, tends to increase to a lesser degree. (The contrast formation mechanism for heavy elements is different, ref ecting simple absorption contrast by thermal scattering out of the bright feld in both $\mathrm{ABF}$ and MaBF imaging.) This approach has been proposed previously in STEM. Assuming aberrated lenses and the weak object approximation, Rose and colleagues showed that lens aberrations lead to rings of destructive and constructive interference between the scattered and unscattered wave, and that optimum signal-to-noise is then achieved by adding the signal from these different regions with different signs $[7,38]$. The method proposed here, which is restricted to atomic resolution, uses the scattering-induced phase shift rather than lens aberrations to generate the contrast. Earlier analyses, being based on the weak phase object approximation, were only clearly valid for very thin samples. The s-state analysis advanced in support of ABF [3, 4] and the Bloch wave analaysis advanced in support of MaBF [5], and by extension eABF imaging as described here, hold over a wide range of thicknesses, as seen in Fig. 2. They also dictate that the division in the diffraction pattern between areas of constructive and destructive interference is determined by the (reciprocal space) width of the column eigenstates, not the aberration function of the probe (which, in aberration-corrected instruments, we strive to keep small), enabling for simpler rules-of-thumb for selection of the inner angle in ABF and the outer angle in MaBF.

An experimental realization of $\mathrm{ABF}, \mathrm{MaBF}$ and eABF imaging for $\mathrm{LaAlO}_{3}$ case is given in Fig. 3. eABF imaging is more difficult to implement than $\mathrm{ABF}$, requiring two separately addressable detectors with gains normalized such that the arithmetic combination of their signals is meaningful. Here we have used the segmented detector of Ref. [39]. However, separate detectors - either a disk and an annular detector, or two disk detectors with one occluding the centre of the other - can also be used. The results of Fig. 3 support the conclusions above: eABF imaging clearly shows improved visibility of the light element columns relative to the heavy element columns when compared against 


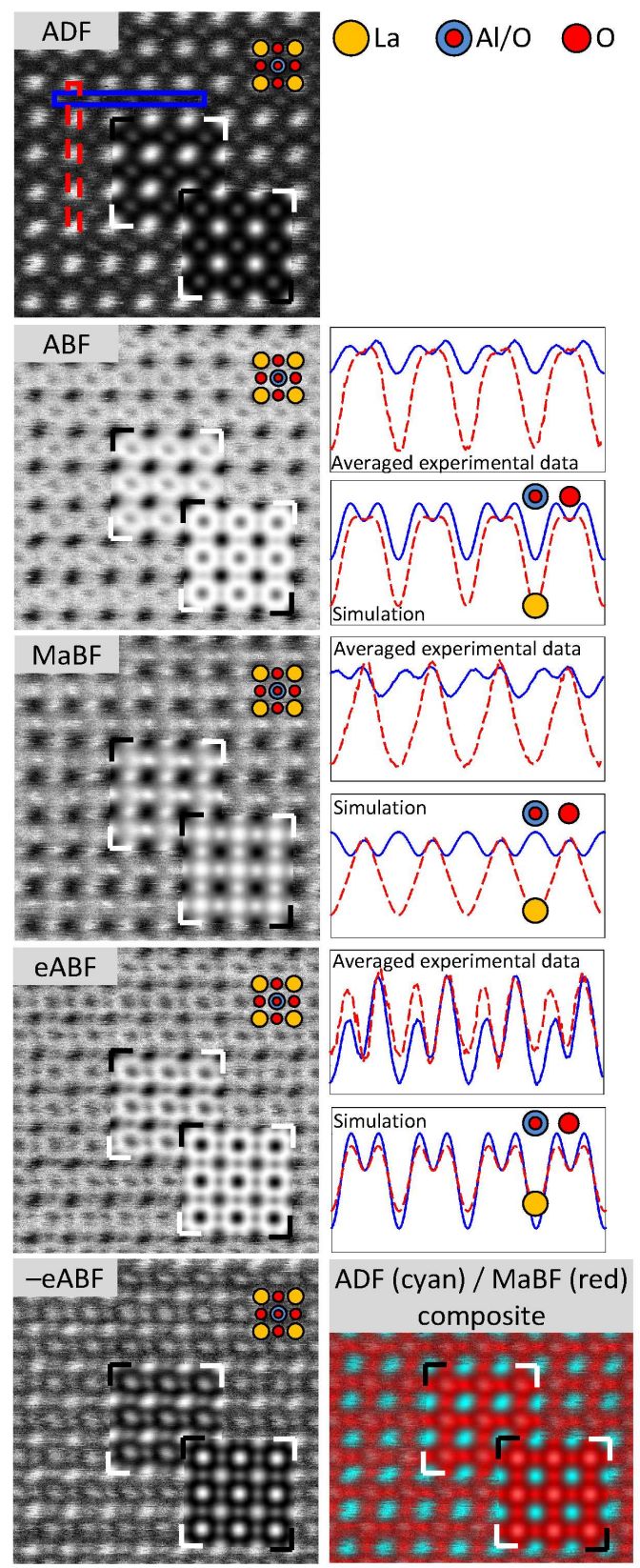

Figure 3: Raw $\mathrm{LaAlO}_{3}$ experimental images recorded on a JEM-2100F TEM/STEM (JEOL Co. Ltd) equipped with an aberration corrector (CEOS $\mathrm{GmbH}$ ), operated at an accelerating voltage of $200 \mathrm{kV}$, and using a segmented detector [39]. Experimental images averaged over the repeating unit, simulated images, and the projected structure are inset. The simulations assume a Gaussian incoherent effective source size of half-width $0.05 \mathrm{~nm}$ but otherwise the same probe-forming and detector collection aperture conditions as per Fig. 2, and, somewhat arbitrarily, a $50 \mathrm{~nm}$ thick specimen. Line scans, taken from regions equivalent to those denoted on the ADF image, are shown for the ABF, MaBF and eABF images for both the averaged experimental images and the simulations. 
$\mathrm{ABF}$, and consistent and direct visual interpretability of all columns when compared against MaBF.

Line scans extracted from the averaged experimental images and the simulated images are shown to the right in Fig. 3 , for both the $\mathrm{La}-\mathrm{O}-\mathrm{La}$ and the $\mathrm{O}-\mathrm{Al} / \mathrm{O}-\mathrm{O}$ column sequences. While the qualitative comparison between experiment and simulation is highly favourable for the $2 \mathrm{D}$ images, these line prof les expose some differences. In particular, some asymmetry appears in the experimental data, most evident in the MaBF and eABF images, suggesting that the crystal is not perfectly on axis. There is also some difference in contrast between the experiment and the simulations, which may ref ect differences in thickness (not measured in the experiment), small mistilt of the sample, the size (and potentially shape [40-43]) assumed to describe the incoherent effective source size, and the use of a pseudo-cubic approximation to the structure which neglects some small structural distortion along the $\mathrm{O}$ column.

The overall sign applied to the eABF image is a matter of taste rather than information content. We have chosen $\mathrm{e} A \mathrm{BF}=\mathrm{ABF}-\mathrm{MaBF}$ because we will mainly focus on the comparison between $\mathrm{eABF}$ and $\mathrm{ABF}$ and this construction gives both the same form of images (i.e. sense of contrast). As an exception, the bottom row in Fig. 3 compares the negative or contrast inverted $\mathrm{ABF}$ image with the $\mathrm{ADF} / \mathrm{MaBF}$ colour composite visualization of Kotaka [6]. The eABF image alone does not, through its contrast, give as clear an indication of the relative column weights, but as regards column visibility and localization is comparable to the colour composite image.

\section{Exploration of the imaging dynamics via simulation}

As always, the strong interaction between probe electrons and the specimen, compounded by the essentially limitless number of different material structures, makes atomic resolution imaging a complicated process and sets the bar high on the quantity and quality of evidence required to demonstrate that an imaging mode is both robust and usefully interpretable. Fortunately, since eABF imaging can be explained as a consequence of the form and behaviour of both $\mathrm{ABF}$ and $\mathrm{MaBF}$ images, the previous explorations of the robustness of $\mathrm{ABF}[3,4]$ and $\mathrm{MaBF}[5]$ can be invoked in support of the new method. Nevertheless, questions about the behaviour of eABF imaging in the presence of residual aberrations and of optimal probe-forming and detector collection angles arise. Concrete evidence supporting our assertion that eABF imaging enhances the SNR for light element columns is also required. In this section we clarify these issues with case-study simulations. We consider three materials: $\mathrm{LaAlO}_{3}$ [001], expanding the explorations already presented; $\mathrm{YH}_{2}$ [001], pushing the limits of the method for the lightest of elements; and $\mathrm{Al}_{3} \mathrm{Li}$ [001], a sample containing only light elements.

In the previous section we used a Gaussian incoherent effective source with $0.05 \mathrm{~nm}$ half-width. This value was not measured experimentally, but rather selected through trial-and-error to produce good visual agreement with experiment, an approach which is tolerably justif ed provided the instrument is otherwise well-characterized [43]. This value is quite broad by the standards of modern instruments in stable environments (see, for example, Ref. [44]), a circumstance we attribute to the slower scan speed used in conjunction with the new segmented detector, and persisting with this value would hamper our explorations of Li- and H-bearing materials. Therefore, unless otherwise 


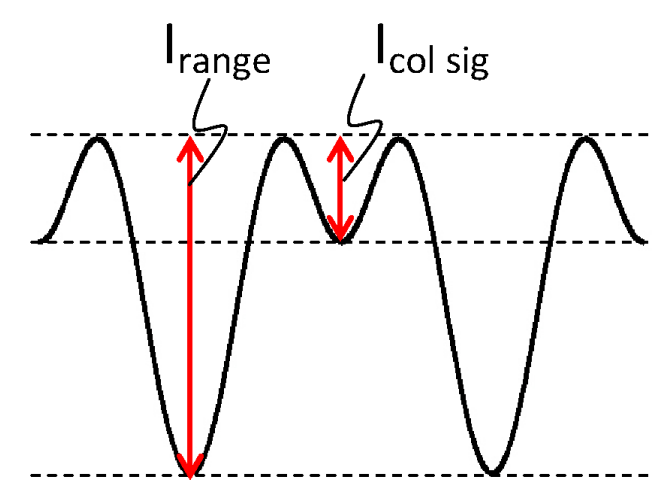

Figure 4: Schematic ABF or eABF prof le (in fact the eABF prof le from Fig. 2(g)) showing how we def ne $I_{\text {col vis, }}$, the local signal of the column, and $I_{\text {range }}$, the intensity range of the image. We def ne a consistent, absolute scale such that the signal $I$ represents a fraction of the total incident intensity (or current).

specif ed, all simulations from this point assume a Gaussian incoherent effective source with $0.04 \mathrm{~nm}$ half-width, which better shows the potential for imaging the lightest of elements whilst remaining a realistic value.

Seeking to discuss quantitative visibility and SNR, it is helpful to def ne a visibility measure. With reference to the example schematic line prof le in Fig. 4, applicable to both ABF and eABF imaging, we def ne the local "signal" of a column of interest, $I_{\mathrm{col} \text { sig }}$, to be the depth of the trough in the signal at that column. To automate the analysis, we assume $I_{\mathrm{col}}$ sig to be equal to the difference between the maximum signal in the image and the signal at the column of interest. In directly interpretable $\mathrm{ABF}$ and $\mathrm{eABF}$ images this is a good approximation, but in images with more anomalous contrast it may be that the maximum image intensity differs appreciably from the intensity rimming the column trough of interest. Inspection of the 2D images is thus a necessary adjunct to drawing conclusions from plots of such single parameter measures. Also shown in Fig. 4, we def ne $I_{\text {range }}$ to be the intensity range of the entire image. The quantity $I_{\text {col sig }} / I_{\text {range }}$, i.e. the signal of interest expressed as a fraction of the image range, is a simple measure of the global contrast/visibility of the column.

A column with low contrast/visibility may be difficult to see in a 2D image. However, this should be clearly distinguished from any statement that the light element column is difficult to detect. The detectability of an individual column is better measured by the SNR of $I_{\text {col sig. }}$. If the SNR is less than unity, f uctuations due to noise are larger than the signal of interest and the detectability of the column is doubtful. For comparison across multiple probe-forming aperture and detection collector sizes, we def ne our noise level to be the shot noise, assuming Poisson statistics, that would be obtained if all the current was collected in a single detector. Appendix A shows how the SNR in $I_{\text {col sig }}$ in $\mathrm{ABF}$ and $\mathrm{eABF}$ is then calculated. We will give $\mathrm{SNR}$ values on the assumption of $5 \%$ noise by the above def nition, but the form of the Eqs. (A.2)-(A.4) makes clear that the SNR varies in proportion to the reference noise level, and so relative SNR comparisons apply more generally.

Mosaics showing how the ABF, MaBF and eABF images vary as a function of thickness and other signif cant 


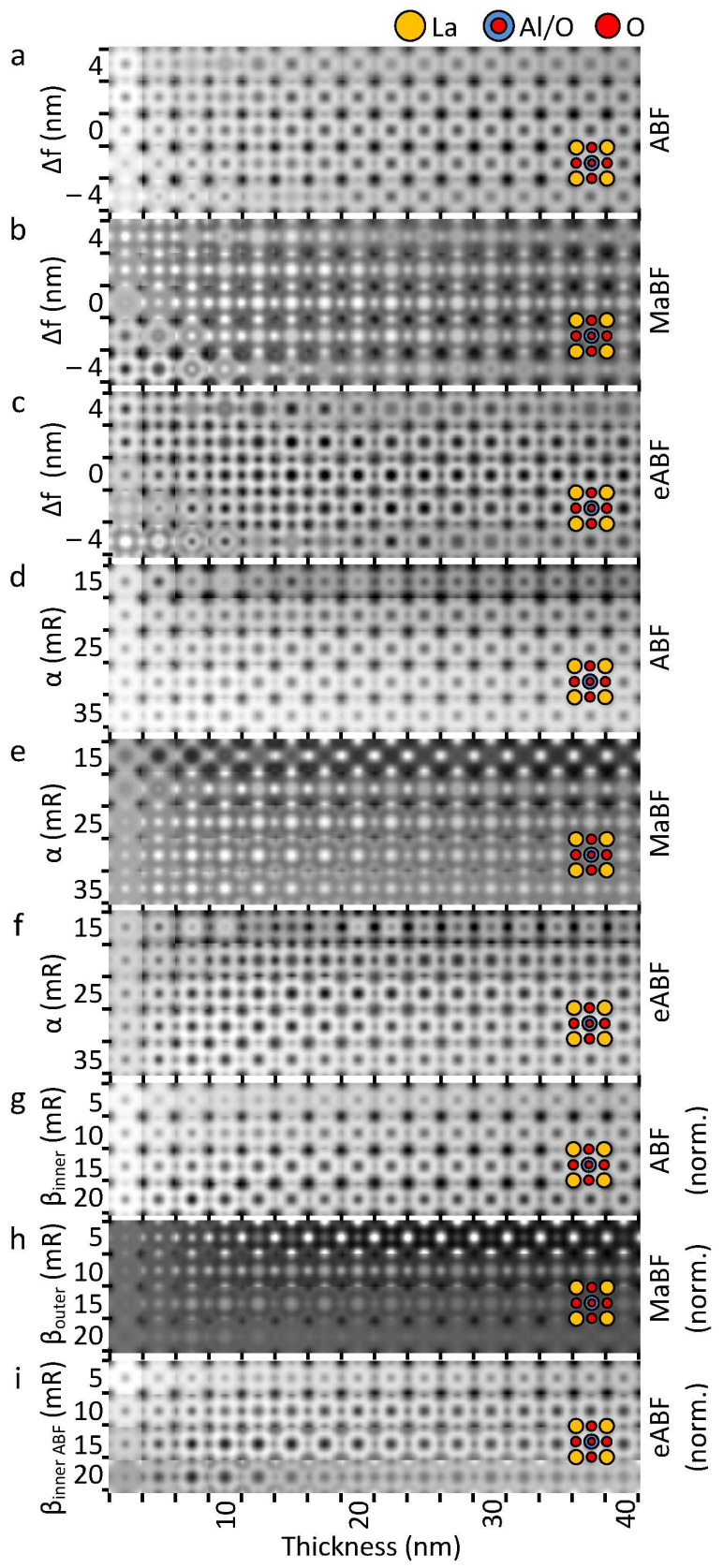

Figure 5: Moasics showing the form of $\mathrm{ABF}, \mathrm{MaBF}$ and $\mathrm{eABF}$ images as a function of thickness and either defocus $(\Delta f)$, probe-forming aperture angle $(\alpha)$ or detector angle $\left(\beta\right.$, either inner or outer as per the subscript and as pertinent to the imaging mode) for a $\mathrm{LaAlO}_{3}$ sample viewed along the [001] zone axis. When $\Delta f$ is varied, $\alpha=23 \mathrm{mrad}$ is assumed, while when $\beta$ is varied, $\alpha=25 \mathrm{mrad}$ is assumed. When either $\Delta f$ or $\alpha$ is varied, the $\mathrm{ABF}$ detector is assumed to span the range $\alpha / 2$ to $\alpha$, while the MaBF detector is assumed to span the range 0 to $\alpha / 2$. When $\beta$ is varied, contrast normalization is applied internal to the mosaic to compensate for the changing detector area. All other mosaics have contrast on an internally consistent scale. 
parameters are given in Fig. 5 for $\mathrm{LaAlO}_{3}$. To supplement the qualitative, appearance-based information in Fig. 5, we plot $I_{\text {col sig }}$ and its SNR for the $\mathrm{O}$ column in $\mathrm{LaAlO}_{3}$ for both $\mathrm{ABF}$ and eABF imaging in Fig. 6 .
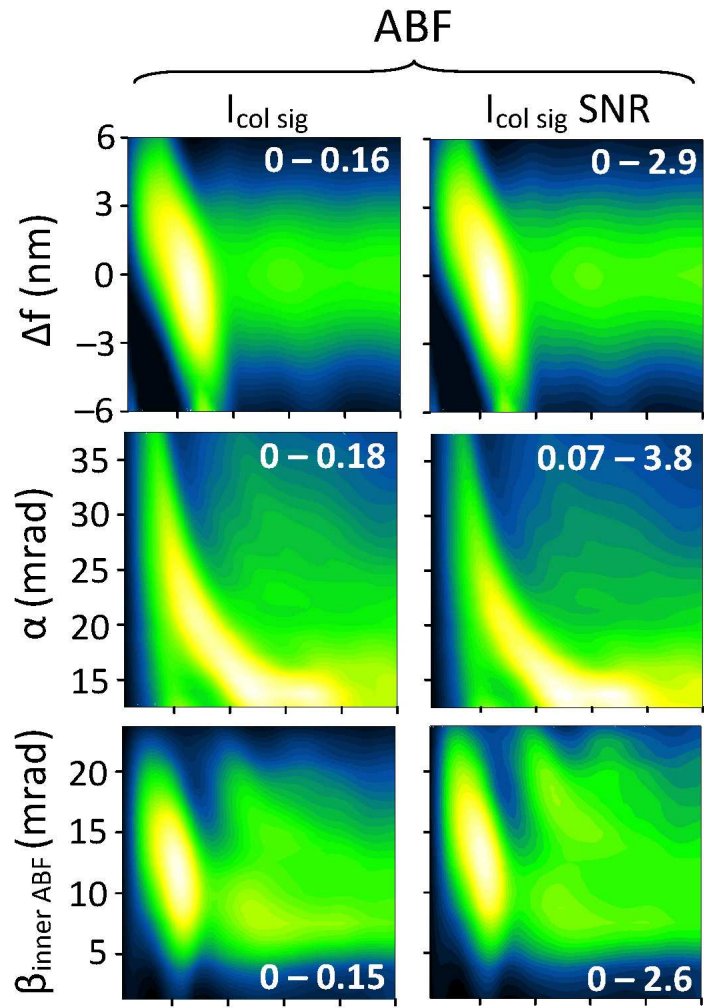

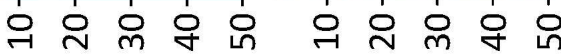
Thickness (nm) Thickness (nm)

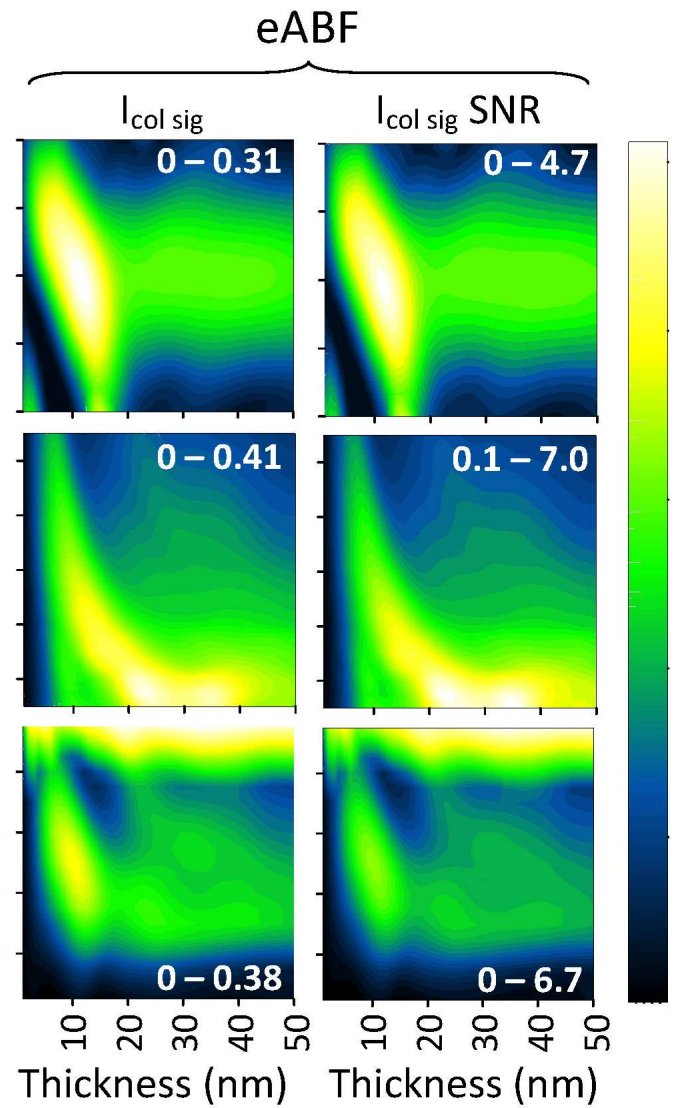

Figure 6: Column signal and SNR maps, for both $\mathrm{ABF}$ and eABF imaging, as a function of thickness and either defocus ( $\Delta f$ ), probe-forming aperture angle $(\alpha)$ or ABF detector inner angle $\left(\beta_{\text {inner ABF }}\right)$ for the pure O columns in $\mathrm{LaAlO}_{3}$ [001]. The superposed numbers describe the range of the images applicable to the common colour bar shown on the right. When $\Delta f$ is varied, $\alpha=23$ mrad is assumed, while when $\beta$ is varied, $\alpha=25$ mrad is assumed. When $\alpha$ is varied, the ABF detector is assumed to span the range $\alpha / 2$ to $\alpha$, while the MaBF detector is assumed to span the range 0 to $\alpha / 2$.

Figs. 5(a)-(c) show mosaics for varying thickness and defocus. A probe-forming aperture of $\alpha=23 \mathrm{mrad}$ is assumed (as used earlier), with the ABF detector spanning the range 11.5-23 mrad and the MaBF detector spanning the range $0-11.5 \mathrm{mrad}$. Figs. 5(d)-(e) show mosaics for varying thickness and probe-forming aperture angle $\alpha$. Here $\Delta f=0 \mathrm{~nm}$ is assumed, with the $\mathrm{ABF}$ detector spanning the range $\alpha / 2$ to $\alpha$ and the MaBF detector spanning the range 0 to $\alpha / 2$. It is assumed that the total current remains the same regardless of probe-forming aperture angle, and this allows for a direct comparison of images across different $\alpha$ values. Figs. 5(g)-(i) show mosaics for varying thickness and detector collection angle. Specif cally, we vary the inner angle of the $\mathrm{ABF}$ detector, $\beta_{\text {inner } \mathrm{ABF}}$, on the assumption that the $\mathrm{ABF}$ detector spans the range $\beta_{\text {inner ABF }}$ to $\alpha$ while the MaBF detector spans the range 0 to $\beta_{\text {inner ABF. For Figs. }}$ 
$5(\mathrm{~g})$-(i), $\alpha=25 \mathrm{mrad}$ is assumed as a conveniently round number (the qualitative difference between $\alpha=23 \mathrm{mrad}$ and $\alpha=25 \mathrm{mrad}$ is negligible), while $\Delta f=0 \mathrm{~nm}$ is again assumed. In order to make comparisons across different detector ranges, the individual tiles in the mosaics in Figs. 5(g) and (h) have been normalized by detector area. This allows for a direct comparison of contrast, but obscures the fact that the absolute signal, and thus more importantly the SNR, is smaller for smaller detector areas. For eABF, the individual tile rows in the mosaics in Fig. 5(i) have been shifted to have zero mean - this preserves the contrast internal to the images while suppressing the uninformative fact that the $\mathrm{eABF}$ signal becomes lower as the portion of electrons in the MaBF detector grows and that in the ABF detector shrinks when increasing the detector angle dividing the two detectors.

All plots in Fig. 6 are normalized to span the full colour range (shown at the right of the fgure); the absolute scale is indicated by the white numbers superposed on each plot. Qualitatively, though, it is worth noting that there is little difference between the column signal plots and the SNR plots for each imaging type. Indeed, there is little difference between the imaging types. Quantitatively, the key point to note is that the ranges are invariably larger for eABF imaging than ABF image: eABF imaging gives a stronger column signal and SNR by almost a factor of two.

In Figs. 5(a)-(c) we $\mathrm{fnd}$ that the ABF, MaBF and eABF images remain interpretable within a range of about $4 \mathrm{~nm}$ either side of just focus, though the contrast at $\pm 4 \mathrm{~nm}$ defocus is notably lower than at $\pm 2 \mathrm{~nm}$ defocus. For very thin crystals, the "optimal" defocus, i.e. that giving the best contrast for the standard image form for each mode, becomes a slight overfocus, as recently explained by Lee et al. [37]. In the top row of Fig. 6, we see that for increasing thickness in the range $0 \mathrm{~nm}$ to about $15 \mathrm{~nm}$, the defocus providing the maximum $I_{\mathrm{col}}$ sig and SNR values varies from slight overfocus to slight underfocus. Beyond about $15 \mathrm{~nm}$, the optimum defocus centres on zero defocus. This shows the transition between the thin thickness limit where coherent lens aberrations play a role [37] and the larger thickness limit where the s-state model and channeling dominate $[4,5]$. There is some visible variation in relative contrast with thickness in Figs. 5(a)-(c). However, excepting the thinnest of crystals, we conclude that the imaging is qualitatively robust over a wide range of thicknesses for an optimal defocus of zero.

In Figs. 5(d)-(f), it can be seen that for most of the probe-forming aperture range shown, the ABF, MaBF and eABF images are reliably interpretable. In the middle row of Fig. 6 we $\mathrm{f}$ nd that the signal at the $\mathrm{O}$ column tends to be greater for smaller probe-forming aperture angles, especially for thicker specimens. This is one case where we should temper our interpretation of these single-measure plots by inspection of the 2D images. Unlike ADF imaging, where the approximate atomic-number-squared contrast applies at all resolutions, $\mathrm{ABF}$ and MaBF are exclusively atomic resolution imaging techniques: the imaging dynamics claimed for these imaging modes are dependent on the probe atop a given column coupling primarily to a single column, and so the imaging breaks down when the probe becomes so broad as to begin to overlap multiple columns. Consequently, smaller aperture sizes, which imply a broader spatial distribution in the electron probe, begin to show "anomalous" features. For instance, Fig. 5(e) shows that the $\mathrm{La} / \mathrm{O}$ column ceases to be visible in MaBF at $\alpha=15 \mathrm{mrad}$, though it appears as a bright spot for $\mathrm{f}$ ner probes. While $\mathrm{ABF}$ and $\mathrm{ABF}$ remain qualitatively interpretable at all thicknesses for $\alpha=15 \mathrm{mrad}$, i.e. all atomic columns appear as troughs, we see more thickness-dependent f uctuation in feature size and contrast at lower probe-forming 
aperture angles than at higher probe-forming aperture angles, and are obliged to conclude that the robustness of the imaging mode is becoming questionable. Because there is an optimal probe-forming angle for coupling to a column [45], very large probe-forming apertures also lead to a reduction of coupling to the column, as evident in the gradual drop off in signal and SNR with increasing probe-forming aperture angle in the middle row of Fig. 6 . This has been discussed previously in the context of $\mathrm{ABF}[4,46]$. Consequently, there is no advantage here to seeking better and better aberration-correction to push for higher and higher probe-forming aperture angles. Because the drop off is gradual, we suggest that $\alpha$ somewhere in the range $20 \mathrm{mrad}$ to $25 \mathrm{mrad}$ is a reasonable compromise between good signal/SNR on the $\mathrm{O}$ columns and robust interpretation of the contrast in the full image.

In Figs. 5(g) and (h), the limits of small ABF inner angle or large MaBF outer angle are the same - a single detector spanning the full bright f eld region, which, being broadly complementary to ADF imaging, is known not to favour light element imaging. Conversely, the limits of large ABF inner angle or small MaBF outer angle correspond to very narrow detectors which can be quite susceptible to coherence effects. Interestingly, though, the case of 5 mrad outer angle for MaBF is seen to make the $\mathrm{O}$ column bright over a wide thickness range. Whereas for $\mathrm{ABF}$ imaging alone an inner angle equal to half the probe-forming aperture angle has been offered previously as a good rule-of-thumb (a conclusion supported here by Fig. 5(g)), high contrast for light elements in MaBF appears to favour a slightly smaller MaBF outer angle (Fig. 5(h)). The consequences of this are most readily apparent in the bottom row of Fig. 6: there is some advantage, slight in $\mathrm{ABF}$ but stronger in $\mathrm{ABF}$, of setting the $\mathrm{ABF}$ detector inner angle (and MaBF detector outer angle) slightly lower than half the probe-forming aperture angle. We would estimate from the f gure that $\beta_{\text {inner ABF }}=\beta_{\text {outer MaBF }} \sim 8 \mathrm{mrad}$ would be a good compromise over a wider range of angles.

We observed before that there is little difference between the imaging types in Fig. 6 . The one exception is the

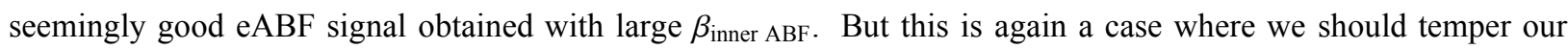
interpretation of these single-measure plots by inspection of the 2D images: close inspection of Fig. 5(i) reveals that the La columns have become bright in the eABF image, meaning that our def nition of $I_{\text {col sig }}$ through reference to the overall image maximum (rather than the intensity rim around the $\mathrm{O}$ column trough) is not a good measure of the local column signal strength.

The broad qualitative conclusions for the $\mathrm{LaAlO}_{3}$ case are seen to hold for the $\mathrm{YH}_{2}$ case in Fig. 7 and the $\mathrm{Al}_{3} \mathrm{Li}$ case in Fig. 8. The broad quantitative conclusions are seen to hold for both cases in Fig. 9 where the SNR of $I_{\text {col sig }}$ is shown for the $\mathrm{H}$ column in $\mathrm{YH}_{2}$ and the $\mathrm{Li}$ column in $\mathrm{Al}_{3} \mathrm{Li}$ for both the $\mathrm{ABF}$ and eABF imaging modes. The optimum defocus over a wide thickness range is close to zero, but slight overfocus is favourable for very thin samples. The $\mathrm{H}$ columns, though, are reliably identif able only for certain thicknesses, most strongly in the vicinity of $10 \mathrm{~nm}$ but more weakly in the vicinity of $32 \mathrm{~nm}$. This suggests coherence effects play a major role, a circumstance which calls into question whether the signal we see on the $\mathrm{H}$ columns is truly indicative of the presence of the $\mathrm{H}$ atoms.

To clarify this, Fig. 10 shows plots of the SNR of $I_{\text {col sig }}$ as a function of thickness and defocus for the nominal $\mathrm{H}$ column site in both the genuine $\mathrm{YH}_{2}$ structure (top row) and also for the related pseudo-structure obtained by removing the $\mathrm{H}$ atoms (bottom row), but with no other consequences for conf guration, spacing or thermal motion of 


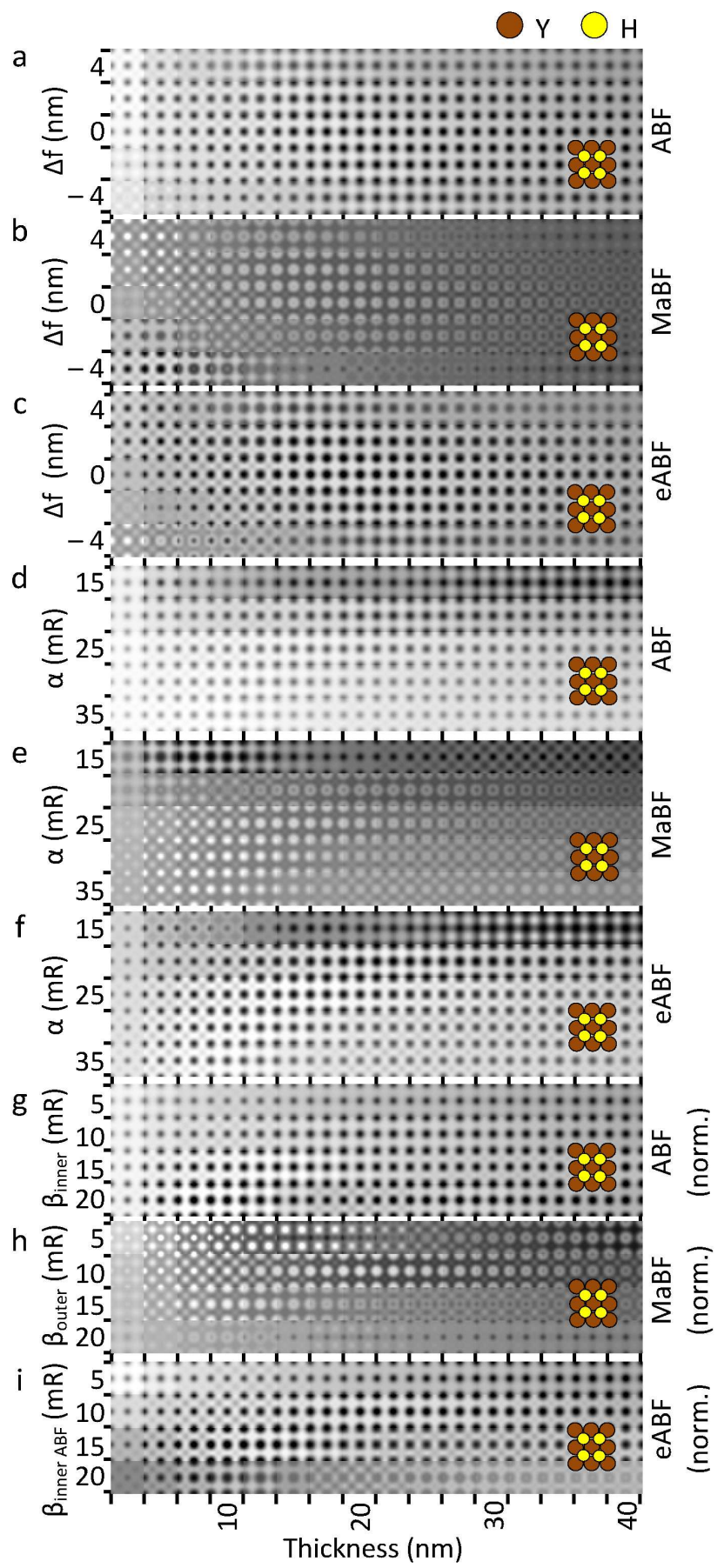

Figure 7: As per Fig. 5, but for a $\mathrm{YH}_{2}$ sample viewed along the [001] zone axis. Structural data obtained from Ref. [47].

the $\mathrm{Y}$ atoms. This f gure also compares the case of perfect spatial coherence $(\sigma=0 \mathrm{~nm}$; left column) with the case including incoherent effective source size ( $\sigma=0.4 \mathrm{~nm}$; right column). This shows $\mathrm{f}$ rstly that most of the suppression 


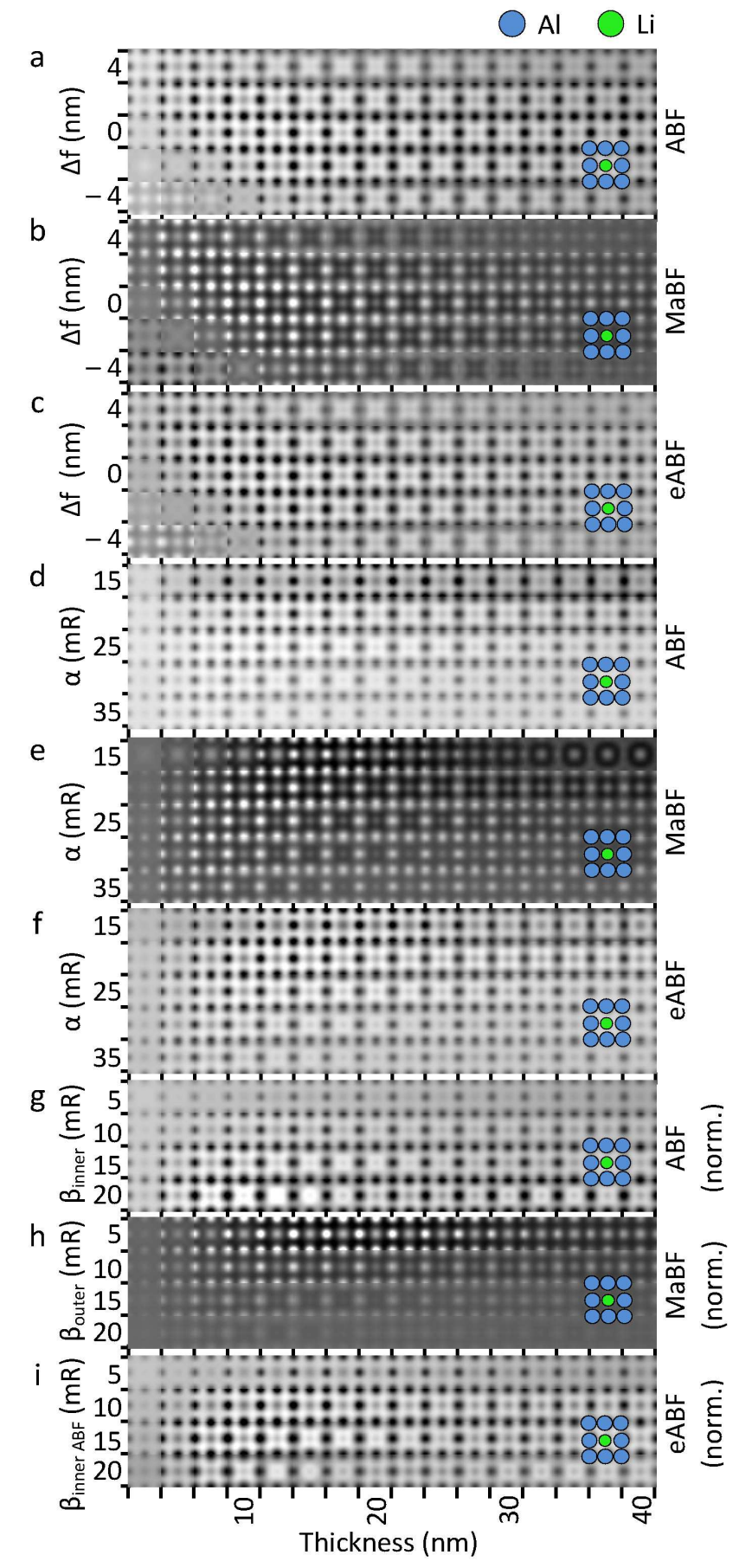

Figure 8: As per Fig. 5, but for an $\mathrm{Al}_{3} \mathrm{Li}$ sample viewed along the [001] zone axis. Structural data obtained from Ref. [48].

of the visibility over different thicknesses is due to the incoherent effective source size, and secondly that the vast majority of the visibility of the trough on the H column location is genuinely due to the presence of the $\mathrm{H}$ atoms, 


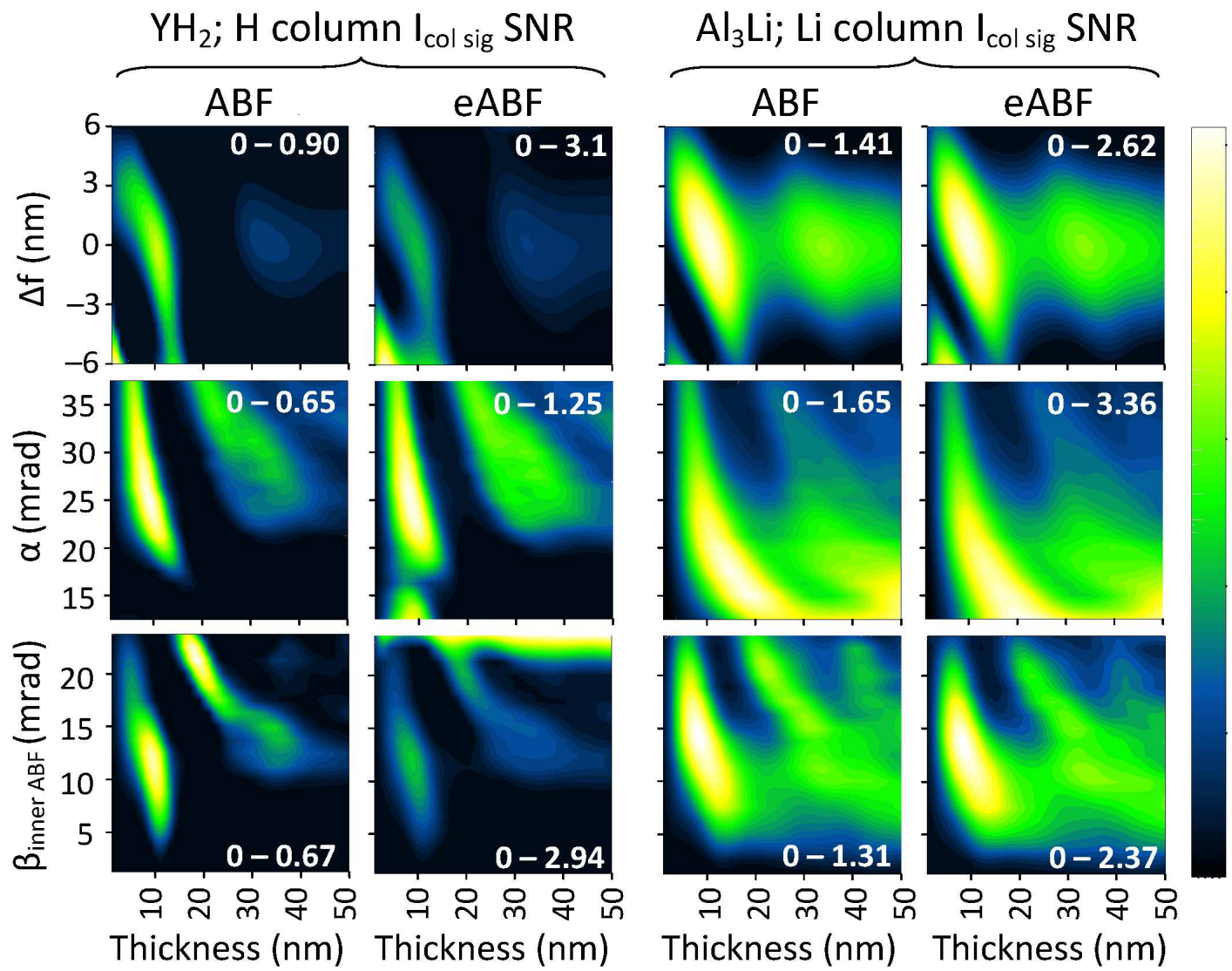

Figure 9: SNR maps of $I_{\mathrm{col} \text { sig }}$ for both ABF and eABF imaging as a function of thickness and either defocus ( $\Delta f$ ), probe-forming aperture angle $(\alpha)$ or $\mathrm{ABF}$ detector inner angle $\left(\beta_{\text {inner } \mathrm{ABF}}\right)$ for $\mathrm{H}$ columns in $\mathrm{YH}_{2}$ [001] (left column pair) and for $\mathrm{Li}$ columns in $\mathrm{Al}_{3} \mathrm{Li}$ [001] (right column pair). The superposed numbers describe the range of the images applicable to the common colour bar shown on the right. When $\Delta f$ is varied, $\alpha=23$ mrad is assumed, while when $\beta$ is varied, $\alpha=25 \mathrm{mrad}$ is assumed. When $\alpha$ is varied, the ABF detector is assumed to span the range $\alpha / 2$ to $\alpha$, while the MaBF detector is assumed to span the range 0 to $\alpha / 2$.

since there is little-to-no signal on that location if they are removed, especially when incoherent effective source size is included.

Returning to Figs. 7 to 9, we f nd that the interpretability can break down for small apertures while the contrast tends to fade for very large apertures. Interestingly, the reliable detection of $\mathrm{H}$ columns seems to favour higher apertures than do most other light elements, though again reliable identif cation is only achieved over certain limited thickness values. If an improvement is to be offered on the rule-of-thumb that the $\mathrm{ABF}$ detector inner angle should be about half the probe-forming aperture angle, it would again, at least in eABF, favour a slightly smaller ABF inner angle / MaBF outer angle since this is seen to favour the visibility of the lighter elements, especially in the case of lithium.

The $\mathrm{H}$ columns in the eABF mosaics for $\mathrm{YH}_{2}$ in Fig. 7 are appreciably more visible than those in the ABF images, 


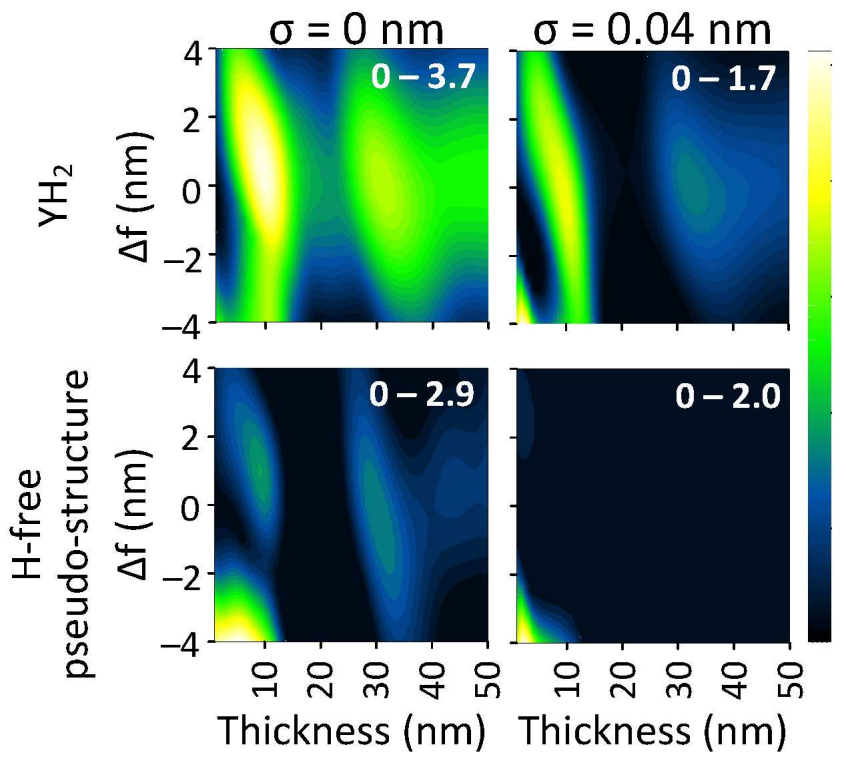

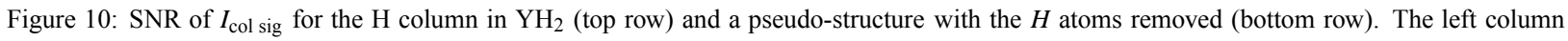
assumes perfect spatial coherence ( $\sigma=0 \mathrm{~nm}$, where $\sigma$ denotes the half width of the assumed-Gaussian incoherent effective source) while the right column incoporates incoherent effective source effects $(\sigma=0.04 \mathrm{~nm})$. The superposed numbers describe the range of the images applicable to the common colour bar shown on the right. $\alpha=23 \mathrm{mrad}$ is assumed.

and this is supported by the quantitative increase in SNR given in Fig. 9. A similar quantitative increase in SNR for eABF over $\mathrm{ABF}$ for the Li columns in $\mathrm{Al}_{3} \mathrm{Li}$ is also attested to by Fig. 9, and one could thus be forgiven for puzzling over why the ABF and eABF mosaics in Fig. 8 are almost indistinguishable. The answer is another reason that we sought to def ne the column signal $I_{\text {col sig }}$ locally rather than with reference to the full image range. $\mathrm{Al}$ and $\mathrm{Li}$ are both light columns, and the eABF construction thus enhances the signal in both column types relative to that of the same columns in ABF. However, the consequence is that there is very little change in relative visibility between the two columns. Fig. 11 seeks to make this clearer. The top row plots the contrast $I_{\text {col sig }} / I_{\text {range }}$ for the Li columns in $\mathrm{Al}_{3} \mathrm{Li}$. Given that the minimum signal in this structure occurs at the Al columns, $I_{\text {range }}$ can also be regarded as the $I_{\text {col sig }}$ signal for the $\mathrm{Al}$ columns. The contrast is thus equivalent to the relative visibility of these two elements, and the top row in Fig. 11 shows very little difference between $A B F$ and eABF for this quantity. This behaviour should be contrasted with the plots for $I_{\mathrm{col}}$ sig and its SNR in, respectively, the middle and bottom rows of Fig. 11, which is basically twice as large in $\mathrm{ABF}$ as it is in ABF. Thus even in cases where the relative visibility is not increased, the SNR and thus column detectability is enhanced by the eABF construction.

\section{Discussion}

As noted previously, constructing the eABF signal requires two independently addressable detectors on which to simultaneously record the $\mathrm{ABF}$ and MaBF signals. In the present implementation, this was accomplished via the 


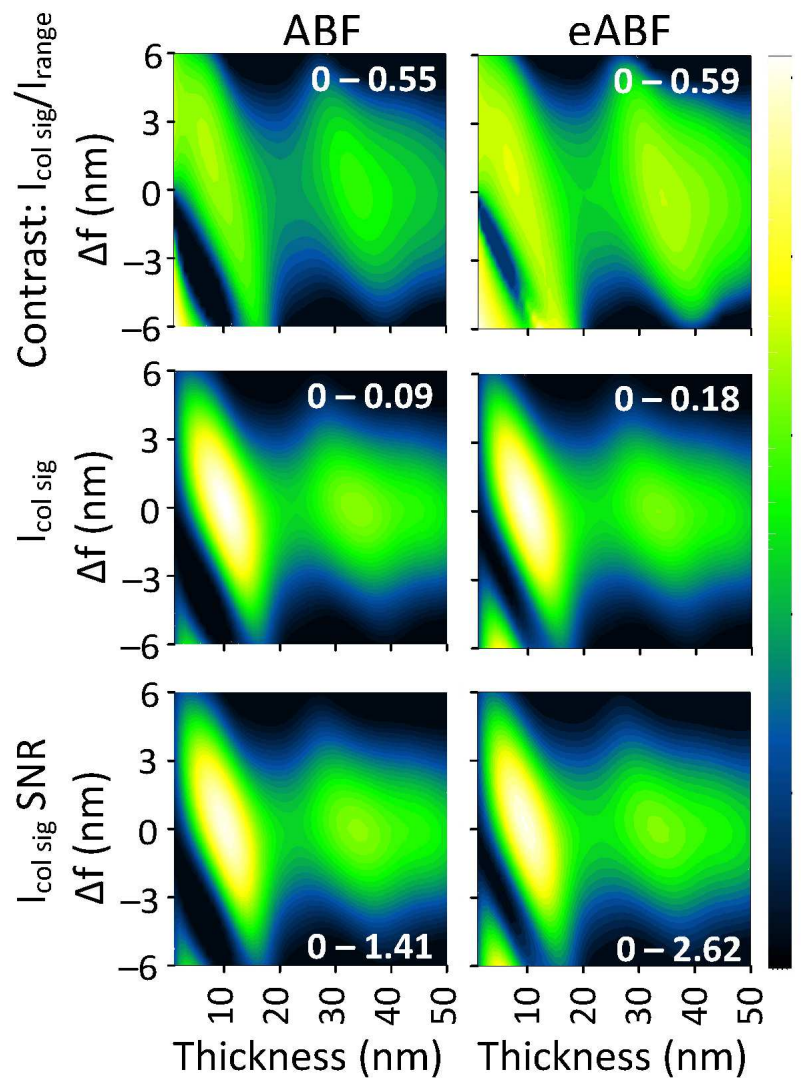

Figure 11: Contrast $\left(I_{\mathrm{col} \text { sig }} / I_{\text {range }}\right)$, signal ( $\left.I_{\text {col sig }}\right)$ and signal SNR maps for the Li columns in $\mathrm{Al}_{3} \mathrm{Li}$ [001], for both $\mathrm{ABF}$ and eABF imaging, as a function of thickness and defocus $(\Delta f)$. The superposed numbers describe the range of the images applicable to the common colour bar shown on the right. $\alpha=23 \mathrm{mrad}$ is assumed.

segmented detector of Ref. [39], where normalization of the signals in the separate segments is part of the standard detector calibration and correct relative centring is assured by the detector construction (up to the uniformity of the detector response). Were eABF to be implemented using either a disk and an annular detector or two disk detectors with one occluding the centre of the other, some care would need to be taken over both the centering and the relative normalization. Any non-uniformity of response across the detectors involved would also have an impact.

Issues relating to centering and non-uniform detector response have recently been explored in detail by Findlay and LeBeau [49]. For detectors in the bright f eld region, signif cant off-centring or non-uniformity (including the effective non-uniformity implied by the shadow cast by the detector support if eABF were implemented using two disk detectors) leads to an observable deviation from the expected rotational symmetry in the images of reasonably-spaced atomic columns. Fig. 12 demonstrates this effect, showing $\mathrm{MaBF}, \mathrm{ABF}$ and eABF images of $\mathrm{LaAlO}_{3}$, comparing the images from perfectly centred detectors against those of detectors with varying degrees of miscentering. A relative miscentering of the detectors is included here, which can only be accomplished in the implementation using two 


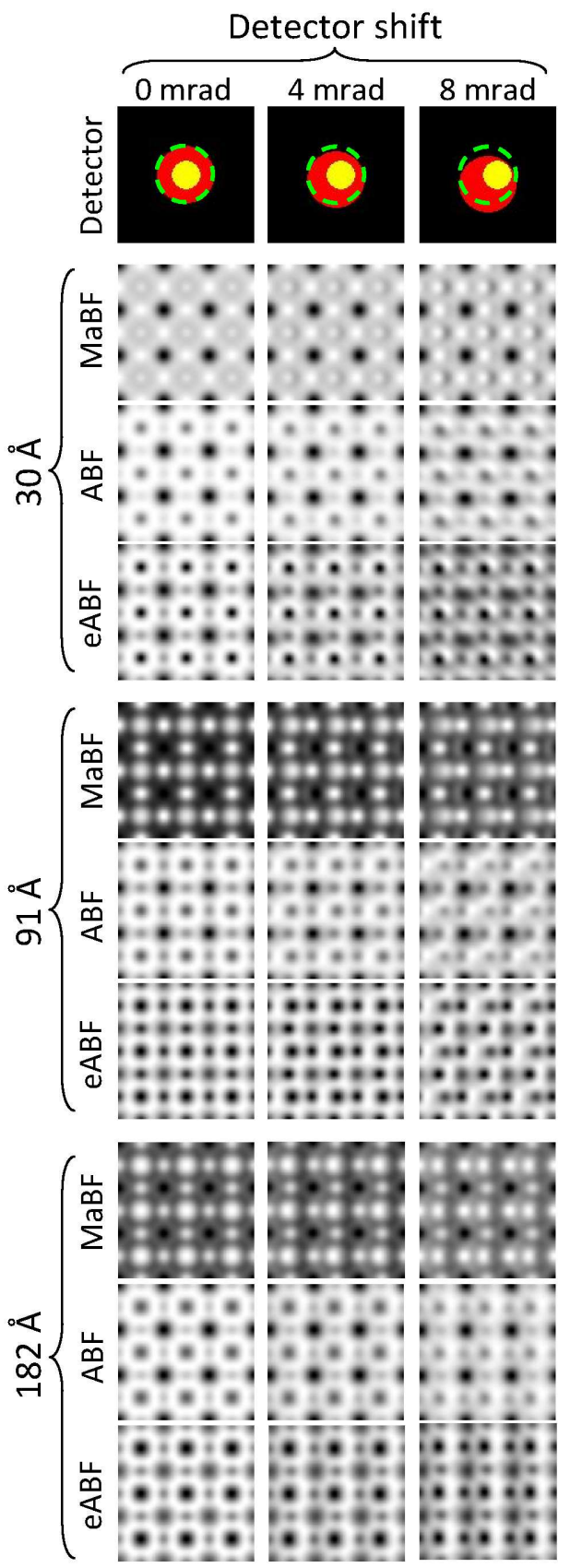

Figure 12: MaBF, $\mathrm{ABF}$ and eABF images of $\mathrm{LaAlO}_{3}$ for different thicknesses, exploring the effect of miscentering the detectors. The $\mathrm{f}$ rst column assumes perfectly centred detectors, while the second and third columns assume displacements of magnitude 4 mrad and 8 mrad, respectively, with the MaBF detector shifted to the right and the $\mathrm{ABF}$ detector down the page. The MaBF detector spans the range $0-11.5 \mathrm{mrad}$ while the $\mathrm{ABF}$ detector spans the range $11.5-23 \mathrm{mrad}$.

overlapping disk detectors (though occlusion due to the support for the upper detector has been neglected). Somewhat surprisingly, the asymmetry is only barely perceptible at $4 \mathrm{mrad}$ miscentering, despite this being a non-trivial fraction 
of the $11.5 \mathrm{mrad}$ radius of the MaBF detector. The incoherent effective source size accounts for part of this - the asymmetry is clearer if perfect coherence is assumed - but the methods appear to be reasonably robust to a small degree of miscentering. By 8 mrad miscentering, the asymmetry is becoming evident in all the images. Bearing in mind that the MaBF and $\mathrm{ABF}$ signals can be inspected individually as well as being combined to produce an eABF image, signif cant off-centering - particularly of the MaBF detector which, being the smaller of the two, is more sensitive - should not go unnoticed.

In the case of implementing eABF using a disk and an annular detector, it would also be possible to inadvertently introduce a gap between the two detectors, i.e. a portion of the bright $f$ eld region covered by neither detector. Such gaps are better thought of as errors in the inner and outer detector angles which, as per Ref. [49], produce problems for quantitative work. For the more semi-quantitative analysis attempted here, however, we again $\mathrm{fnd}$ that it is only if the gaps are quite large that we may start to see anomalous features.

We propose $\mathrm{ABF}$ imaging as a means to produce an image with better signal-to-noise for light elements than the $\mathrm{ABF}$ image and better visual interpretability than the MaBF image. This is particularly useful for real-time imaging of samples containing very light elements. It is worth emphasizing, though, that the MaBF and ABF images obtained as part of forming the eABF image can be inspected individually (as can any other simultaneously acquired signal, for instance $\mathrm{ADF}$ ). In making light elements more visible by reducing the relative contrast between light and heavy element columns, eABF necessarily sacrif ces discriminating power between columns of differing compositions. Information from the $\mathrm{ADF}$ and MaBF images should be used in such cases to better interpret the composition of the sample.

We stated earlier that correct relative normalization between the two detectors was important. Since the consequence of incorrect normalization will simply be to bias the $\mathrm{ABF}-\mathrm{MaBF}$ difference image to favour either the $\mathrm{ABF}$ or the (contrast inverted) MaBF image, normalization errors are not so much likely to produce misleading results remember, the MaBF and $\mathrm{ABF}$ images can also be analyzed individually - as they are to forego either the signal-tonoise ratio or the direct interpretability advantages of eABF. It may even be that, at least in some cases, a carefully weighted difference would offer still further improvements relative to either MaBF or ABF, but any controlled attempt would necessarily proceed from a knowledge of the correct relative normalization.

Finally, we note that eABF imaging inherits all the limitations previously established for ABF imaging [4]. In particular, the method is reliant on having straight and reasonably well-separated columns along which the electron probe can channel. Therefore, though the analysis may benef $t$ from multiple, simultaneously acquired images, we do not expect eABF to be well-suited to samples involving a signif cant concentation of vacancies, strain-induced static atomic displacements which vary along the beam direction or other such defects. 


\section{Conclusion}

We have shown that by taking the difference between STEM images formed with electrons in the outer portion of the bright $f$ eld region, i.e. the $\mathrm{ABF}$ image, and the inner portion of the bright $\mathrm{f}$ eld region, i.e. the MaBF image, that we obtain an image with direct and robust interpretability comparable to ABF but with improved detectability of the light element columns. This improved detectability may, in images from specimens containing both light and heavy elements, manifest in a qualitative improvement of the visibility of the light elements relative to the heavy elements. Generally, even in images from specimens containing only light elements, it manifests as an improvement in the signal-to-noise ratio of all light element columns. Though utilizing two detectors (ABF and MaBF) with correct relative normalization of signals is appreciably more involved than obtaining either image alone, we consider that eABF nevertheless constitutes a worthwhile advance in the quest to image light elements, a goal that remains challenging for the very lightest of elements and/or in the presence of non-trivial incoherent effective source size. As a prescription for arithmatic combination of signal from different regions of the diffraction pattern, it also represents one suggestion about how greater sensitivity, measured by SNR, can be obtained from processing-here in hardware but ultimately in software when full diffraction patterns are recorded [50]-the full electron scattering distribution.

\section{Appendix A. Signal-to-noise ratio}

Assuming Poisson statistics, the mean signal $\mu$ at any probe point is related to the standard deviation in the signal $\sigma$ at that same probe point via $\sigma^{2}=\mu$. The noise level $\tilde{n}$, expressed as a fraction, is thus related to the mean signal as per:

$$
\tilde{n} \equiv \frac{\sigma}{\mu}=\frac{1}{\sqrt{\mu}} .
$$

For instance, a $5 \%$ noise level $(\tilde{n}=0.05)$ requires a mean signal of $\mu=400$.

For the purposes of determining shot noise, all probe positions are completely independent of one another. Let us set a reference noise level $\tilde{n}_{\text {ref }}$ determined via Eq. (A.1) by the mean signal $\mu_{\text {ref }}$ obtained when all the current in the probe is collected on a single detector. By our convention for intensity normalization, the associated intensity $I_{\text {ref }}=1$. An intensity $I^{\prime}$ recorded on some detector for some probe position will thus have a shot noise of

$$
\tilde{n}^{\prime}=\frac{1}{\sqrt{\mu_{\text {ref }} \times I^{\prime} / I_{\text {ref }}}}=\frac{\tilde{n}_{\text {ref }}}{\sqrt{I^{\prime}}} .
$$

It readily follows by standard propagation-of-errors analysis that the SNR of $I_{\mathrm{vis}}$ col in an ABF image is given by

$$
\mathrm{SNR}_{\mathrm{ABF}}=\frac{I_{\mathrm{vis} \text { col ABF }}}{\Delta I_{\mathrm{vis} \text { col ABF }}}=\frac{I_{\max \mathrm{ABF}}-I_{\mathrm{col} \mathrm{ABF}}}{\tilde{n}_{\mathrm{ref}} \sqrt{I_{\max \mathrm{ABF}}+I_{\mathrm{col} \mathrm{ABF}}}},
$$


since the error in $I_{\mathrm{vis} \mathrm{col} \mathrm{ABF}}$ is given by $\Delta I_{\mathrm{vis}}$ col ABF $=\sqrt{\left(\tilde{n}_{\max \mathrm{ABF}} I_{\max \mathrm{ABF}}\right)^{2}+\left(\tilde{n}_{\mathrm{col} \mathrm{ABF}} I_{\mathrm{col} \mathrm{ABF}}\right)^{2}}$. Likewise, for eABF

$$
\begin{aligned}
\mathrm{SNR}_{\mathrm{eABF}} & =\frac{I_{\mathrm{vis}} \text { col eABF }}{\Delta I_{\mathrm{vis} \mathrm{col} \mathrm{eABF}}} \\
& =\frac{\left[I_{\mathrm{ABF}}^{*}-I_{\mathrm{MaBF}}^{*}\right]-\left[I_{\mathrm{col} \mathrm{ABF}}-I_{\mathrm{col} \mathrm{MaBF}}\right]}{\tilde{n}_{\mathrm{ref}} \sqrt{I_{\mathrm{ABF}}^{*}+I_{\mathrm{MaBF}}^{*}+I_{\mathrm{col} \mathrm{ABF}}+I_{\mathrm{col} \mathrm{MaBF}}}},
\end{aligned}
$$

where $I_{\mathrm{ABF} / \mathrm{MaBF}}^{*}$ denotes the intensity in the $\mathrm{ABF} / \mathrm{MaBF}$ image at the pixel at which the eABF intensity is a maximum.

\section{Acknowledgements}

We thank N. Saito and K. Nayuki for their assistance in specimen preparation and image processing. This research was supported under the Discovery Projects funding scheme of the Australian Research Council (Project No. DP110101570). This work was also supported by the PRESTO, JST and JSPS KAKENHI Grant number 23686093. A part of this work was also supported by JSPS through its FIRST Program. Part of this work was conducted in Research Hub for Advanced Nano Characterization, The University of Tokyo, supported by MEXT, Japan.

\section{References}

\section{References}

[1] S. J. Pennycook, D. E. Jesson, Ultramicroscopy 37 (1991) 14-38.

[2] E. Okunishi, I. Ishikawa, H. Sawada, F. Hosokawa, M. Hori, Y. Kondo, Microsc. Microanal. 15 (Suppl 2) (2009) 164.

[3] S.D. Findlay, N. Shibata, H. Sawada, E. Okunishi, Y. Kondo, T. Yamamoto, Y. Ikuhara, Appl. Phys. Lett. 95 (2009) 191913.

[4] S.D. Findlay, N. Shibata, H. Sawada, E. Okunishi, Y. Kondo, Y. Ikuhara, Ultramicroscopy 110 (2010) 903-923.

[5] M. Ohtsuka, T. Yamazaki, Y. Kotaka, I. Hashimoto, K. Watanabe, Ultramicroscopy 120 (2012) 48-55.

[6] Y. Kotaka, Appl. Phys. Lett. 101 (2012) 133107.

[7] H. Rose, Optik 39 (1974) 416-436.

[8] J. M. Cowley, Ultramicroscopy 49 (1993) 4-13.

[9] M. Hammel, H. Rose, Ultramicroscopy 58 (1995) 403-415.

[10] G. Radtke, M. Couillard, G.A. Botton, D. Zhu, C.J. Humphreys, Appl. Phys. Lett. 97 (2010) 251901.

[11] H. Hojo, T. Mizoguchi, H. Ohta, S.D. Findlay, N. Shibata, T. Yamamoto, Y. Ikuhara, Nano Letters 10 (2010) 4668-4672.

[12] M. Haruta, H. Kurata, K. Matsumoto, S. Inoue, Y. Shimakawa, S. Isoda, J. Appl. Phys. 110 (2011) 033708.

[13] T. Hosokura, N. Iwaji, T. Nakagawa, A. Ando, H. Takagi, Y. Sakabe, K. Hirao, Crystal Growth and Design 11 (2011) 4253-4256.

[14] A.A. Belik, A.M. Abakumov, A.A. Tsirlin, J. Hadermann, J. Kim, G. Van Tendeloo, E. Takayama-Muromachi, Chem. Mater. 23 (2011) 4505-4514.

[15] S. Kobayashi, S.D. Findlay, N. Shibata, T. Mizoguchi, Y. Sato, E. Okunishi, Y. Ikuhara, T. Yamamoto, Appl. Phys. Lett. 100 (2012) 193112.

[16] S. Zheng, C.A.J. Fisher, T. Kato, Y. Nagao, H. Ohta, Y. Ikuhara, Appl. Phys. Lett. 101 (2012) 191602.

[17] W. Dachraoui, J. Hadermann, A.M. Abakumov, A.A. Tsirlin, D. Batuk, K. Glazyrin, C. McCammon, L. Dubrovinsky, G. Van Tendeloo, Chem. Mater. 24 (2012) 1378-1385.

[18] X.K. Wei, T. Zou, F. Wang, Q.H. Zhang, Y. Sun, L. Gu, A. Hirata, M.W. Chen, Y. Yao, C.Q. Jin, R.C. Yu, J. Appl. Phys. 111 (2012) 073904. 
[19] M. de la Mata, C. Magen, J. Gazquez, M.I.B. Utama, M. Heiss, S. Lopatin, F. Furtmayr, C.J. Fernandez-Rojas, B. Peng, J.R. Morante, R. Rurali, M. Eickhoff, A. Fontcuberta i Morral, Q. Xiong, J. Arbiol, Nano Lett. 12 (2012) 2579-2586.

[20] Z. Xu, K. Jin, L. Gu, Y. Jin, C. Ge, C. Wang, H. Guo, H. Lu, R. Zhao, G. Yang, Small 8 (2012) 1279-1284.

[21] Z. Wang, L. Gu, M. Saito, S. Tsukimoto, M. Tsukada, F. Lichtenberg, Y. Ikuhara, J.G. Bednorz, Adv. Mater. 25 (2013) 218-222.

[22] Y. Oshima, H. Sawada, F. Hosokawa, E. Okunishi, T. Kaneyama, Y. Kondo, S. Niitaka, H. Takagi, Y. Tanishiro, K. Takayanagi, J. Electron Microscopy 59 (2010) 457-461.

[23] R. Huang, Y.H. Ikuhara, T. Mizoguchi, S.D. Findlay, A. Kuwabara, C.A.J. Fisher, H. Moriwake, H. Oki, T. Hirayama, Y. Ikuhara, Agnew. Chem. Int. Ed. 50 (2011) 3053-3057.

[24] R. Huang, T. Hitosugi, S.D. Findlay, C.A.J. Fisher, Y.H. Ikuhara, H. Moriwake, H. Oki, Y. Ikuhara, Appl. Phys. Lett. 98 (2011) 051913.

[25] L. Gu, C. Zhu, H. Li, Y. Yu, C. Li, S. Tsukimoto, J. Maier, Y. Ikuhara, J. Am. Chem. Soc. 133 (2011) 4661-4663.

[26] X. He, L. Gu, C. Zhu, Y. Yu, C. Li, Y.-S. Hu, H. Li, S. Tsukimoto, J. Maier, Y. Ikuhara, X. Duan, Mater. Express 1 (2011) 43-50.

[27] S. Lee, Y. Oshima, H. Sawada, F. Hosokawa, E. Okunishi, T. Kaneyama, Y. Kondo, S. Niitaka, H. Takagi, Y. Tanishiro, K. Takayanagi, J. Appl. Phys. 109 (2011) 113530.

[28] L. Suo, W. Han, X. Lu, L. Gu, Y.-S. Hu, H. Li, D. Chen, L. Chen, S. Tsukimoto, Y. Ikuhara, Phys. Chem. Chem. Phys. 14 (2012) $5363-5367$.

[29] X. Lu, Y. Sun, Z. Jian, X. He, L. Gu, Y.-S. Hu, H. Li, Z. Wang, W. Chen, X. Duan, L. Chen, J. Maier, S. Tsukimoto, Y. Ikuhara, Nano Letters 12 (2012) 6192-6197.

[30] X. Lu, L. Zhao, X. He, R. Xiao, L. Gu, Y.-S. Hu, H. Li, Z. Wang, X. Duan, L. Chen, J. Maier, Y. Ikuhara, Adv. Mater. 24 (2012) $3233-3238$.

[31] S.D. Findlay, T. Saito, N. Shibata, Y. Sato, J. Matsuda, K. Asano, E. Akiba, T. Hirayama, Y. Ikuhara, Appl. Phys. Expr. 3 (2010) 116603.

[32] R. Ishikawa, E. Okunishi, H. Sawada, Y. Kondo, F. Hosokawa, E. Abe, Nature Materials 10 (2011) 278-281.

[33] H. Rose, J. Fertig, Ultramicroscopy 2 (1976) 77-87.

[34] P. Geuens, D. Van Dyck, Adv. Imaging Electron Phys. 136 (2005) 111-226.

[35] B.C. Chakoumakos, D.G. Schlom, M. Urbanik, J. Luine, J. Appl. Phys. 83 (1998) 1979-1982.

[36] P.D. Nellist, J.M. Rodenburg, Ultramicroscopy 54 (1994) 61-74.

[37] S. Lee, Y. Oshima, E. Hosono, H. Zhou, K. Takayanagi, Ultramicroscopy 125 (2013) 43-48.

[38] M. Hammel, H. Rose, Ultramicroscopy 58 (1995) 403-415.

[39] N. Shibata, Y. Kohno, S.D. Findlay, H. Sawada, Y. Kondo, Y. Ikuhara, J. Electron Microscopy 59 (2010) 473-479.

[40] C. Dwyer, R. Erni, J. Etheridge, Ultramicroscopy 110 (2010) 952-957.

[41] C. Maunders, C. Dwyer, P.C. Tiemeijer, J. Etheridge, Ultramicroscopy 111 (2011) 1437-1446.

[42] J. Verbeeck, A. Béché, W. Van den Broek, Ultramicroscopy 120 (2012) 35-40.

[43] C. Dwyer, C. Maunders, C. L. Zheng, M. Weyland, P.C. Tiemeijer, J. Etheridge, Appl. Phys. Lett. 100 (2012) 191915.

[44] S. Kim, Y. Oshima, H. Sawa, T. Kaneyama, Y. Kondo, M. Takeguchi, Y. Nakayama, Y. Tanishiro, K. Takayanagi, J. Electron Microscopy 60 (2011) 109-116.

[45] Y. Peng, P.D. Nellist, S.J. Pennycook, Ultramicroscopy 53 (2004) 257-266.

[46] S.D. Findlay, N.R. Lugg, N. Shibata, L.J. Allen, Y. Ikuhara, Ultramicroscopy 111 (2011) 1144-1154.

[47] D. Khatamian, W.A. Kamitakahara, R.G. Barnes, D.T. Peterson, Phys. Rev. B 21 (1980) 2622-2624.

[48] Z. Li, J.S. Tse, Phys. Rev. B 61 (2000) 14531.

[49] S.D. Findlay, J.M. LeBeau, Ultramicroscopy 124 (2013) 52-60.

[50] K. Kimoto, K. Ishizuka, Ultramicroscopy 111 (2011) 1111-1116. 